\title{
NACO-SDI imaging of known companion host stars from the AAPS and Keck planet search surveys
}

\author{
J. S. Jenkins ${ }^{1}$, H. R. A. Jones ${ }^{2}$, B. Biller ${ }^{3}$, S. J. O’ Toole ${ }^{4}$, D. J. Pinfield ${ }^{2}$, L. Close ${ }^{5}$, C. G. Tinney ${ }^{6}$, R. P. Butler ${ }^{7}$ \\ R. Wittenmyer ${ }^{6}$, B. Carter $^{8}$, and A. C. Day-Jones ${ }^{1}$ \\ 1 Department of Astronomy, Universidad de Chile, Casilla 36-D, Santiago, Chile \\ e-mail: jjenkins@das.uchile.cl* \\ 2 Center for Astrophysics, University of Hertfordshire, College Lane Campus, Hatfield, Hertfordshire, UK, AL10 9AB, UK \\ 3 Institute of Astronomy, 2680 Woodlawn Drive, Honolulu, HI 96822, USA \\ 4 Anglo-Australian Observatory, PO Box 296, Epping 1710, Australia \\ 5 Steward Observatory, University of Arizona, Tucson, AZ 85721, USA \\ ${ }^{6}$ Department of Astrophysics \& Optics, University of New South Wales, NSW 2052, Australia \\ 7 Carnegie Institute of Washington, Department of Terrestrial Magnetism, 5241 Broad Branch Road NW, Washington, \\ DC 20015-1305, USA \\ 8 Faculty of Sciences, University of Southern Queensland, Toowoomba, 4350, Australia
}

Received 25 November 2009 / Accepted 2 March 2010

ABSTRACT

\begin{abstract}
Context. Direct imaging of brown dwarfs as companions to solar-type stars can provide a wealth of well-constrained data to "benchmark" the physics of such objects, since quantities like metallicity and age can be determined from their well-studied primaries. Aims. We present results from an adaptive optics imaging program on stars drawn from the Anglo-Australian and Keck Planet Search projects, with the aim of directly imaging known cool companions.

Methods. Simulations have modeled the expected contrast ratios and separations of known companions using estimates of orbital parameters available from current radial-velocity data and then a selection of the best case objects were followed-up with high contrast imaging to attempt to directly image these companions.

Results. These simulations suggest that only a very small number of radial-velocity detected exoplanets with consistent velocity fits and age estimates could potentially be directly imaged using the VLT's Simultaneous Differential Imaging system and only under favorable conditions. We also present detectability confidence limits from the radial-velocity data sets and show how these can be used to gain a better understanding of these systems when combined with the imaging data.

For HD32778 and HD91204 the detectabilities help little in constraining the companion and hence almost all our knowledge is drawn from the SDI images. Therefore, we can say that these stars do not host cool methane objects, out to on-sky separations of $\sim 2$ ", with contrasts less than 10-11 mag. However, for HD25874, HD120780 and HD145825, the contrasts and detectabilities can rule out a number of possible solutions, particularly at low angular separations, and for the best case, down to strong methane masses of $40 M_{\mathrm{J}}$ at $1^{\prime \prime}$ separation. The contrast curves constructed for these five stars show $5 \sigma$ contrasts $(\Delta \mathrm{F} 1)$ of $\sim 9.2-11.5$ mag at separations of $\geq 0.6^{\prime \prime}$, which correspond to contrasts of $\sim 9.7-12.0$ mag for companions of mid-T spectral type. Such limits allow us to reach down to $40 M_{\mathrm{J}}$ around fairly old field dwarfs that typically constitute high precision radial-velocity programs. Finally, the analysis performed here can serve as a template for future projects that will employ extreme-AO systems to directly image planets already indirectly discovered by the radial-velocity method.
\end{abstract}

Key words. binaries: general - binaries: spectroscopic - planetary systems - stars: imaging - technique: radial velocities techniques: high angular resolution

\section{Introduction}

The detection of over 400 planets orbiting Sun-like stars has revolutionised our knowledge of our local neighbourhood and our position therein. Yet planets are not the sole close companions to solar-type stars. For instance, Duquennoy \& Mayor (1991a,b) and Duquennoy et al. (1992) have examined stellar multiplicity in a series of papers. Radial-velocity surveys have revealed few brown dwarfs orbiting solar-type stars (e.g. Wittenmyer et al. 2009; Jenkins et al. 2009) leading to the phrase "brown dwarf desert" being coined to describe this paucity (Marcy \& Butler 2000). However, beyond 4 AU one would expect few radial-velocity planetary or brown dwarf companions

* Based on observations made with the ESO telescopes at the La Silla Paranal observatory under program ID 076.C-0877(B). to be known due to the limited temporal coverage at the required precision levels necessary to fully sample such companions. In addition, radial-velocity surveys also have strong biases against the detection of long-period companions, as the radial-velocity amplitude is a strong function of orbital period and also since this technique requires the observation of at least half an orbit (e.g. Wright et al. 2007) to constrain companion properties. Only now are we sensitive enough to detect solar system-like gas giant planets in solar system-like orbits (e.g. Jones et al. 2010).

Conversely, direct and coronographic imaging techniques can probe much wider separations than current radial-velocity programs can reach. For example, Kalas et al. (2008) and Marois et al. (2008) have directly imaged planetary mass companions to the stars Fomalhaut and HR 8799, located at angular separations of $14.9^{\prime \prime}$ and $1.73^{\prime \prime}$, or $115 \mathrm{AU}$ and $68 \mathrm{AU}$, respectively. 
McCarthy \& Zuckerman (2004) found another deficit of brown dwarf companions between 75-1200 AU. Liu et al. (2002) used the Gemini-North and Keck Adaptive Optics (AO) systems to obtain three epochs of images of the brown dwarf companion to HR 7672, which had initially been detected by its radial-velocity signature. The flux ratio at $2.16 \mu \mathrm{m}$ was found to be $8.6 \mathrm{mag}$ at a separation of $0.79^{\prime \prime}$. This level of contrast pushed the instrumentation used in this detection to its very limits. However the introduction of Simultaneous Differential Imaging (SDI) on the VLT's NACO facility permits the achievement of higher contrasts, at smaller separations, for the coolest stellar companions. For example, contrasts on the order of $\Delta H \sim 13$ have been demonstrated at $\sim 0.5^{\prime \prime}$ by Mugrauer \& Neuhäuser (2005) and Biller et al. (2007).

\section{Target selection}

In order to guide the selection of target host stars for adaptive optics imaging of brown dwarfs and exoplanets, we have performed simulations which take the best currently available companion parameters from radial-velocity data sets, combined with host-star age estimates and brown dwarf and exoplanetary interior models, to derive predicted magnitude differences and angular separations on sky. These simulations were performed for all stars in the Anglo-Australian and Keck Planet Searches (for samples see Jones et al. 2002; Marcy et al. 2005; Butler et al. 2006 and references therein), which show a long term radial-velocity profile consistent with an orbiting low-mass companion.

\subsection{Angular separation}

Hipparcos distance data (van Leeuwen \& Fantino 2005) is available for all these objects (which all lie at distances of less than $100 \mathrm{pc}$ ). It should be noted that in most cases, the radial-velocity orbital solutions are not well constrained. This is largely because the companion orbits are much longer than the monitoring baselines of the surveys, and in some cases because the companion properties have been derived with no inflection in the radial-velocity curve (often referred to in the planet searches as a "liner"). The fits to both these classes of data produce only semi-major axis lower limits. In addition, the eccentricities of most of the companions are so poorly constrained that they are fixed to zero, causing further separation ambiguity.

\subsection{Contrast ratio}

Infrared photometry for the primary stars were taken from the 2MASS catalogue (http: //irsa. - ipac . caltech.edu/) and when combined with Hipparcos distances we were able to generate accurate absolute $J H K_{\mathrm{s}}$ magnitudes. The $K_{\mathrm{s}}$ from $2 \mathrm{MASS}$ was converted to $\mathrm{K}$ using the magnitude corrections in Carpenter (2001). Absolute magnitudes for the companions were estimated using the non-grey evolutionary tracks of Burrows et al. (1997), the COND models (Baraffe et al. 2003) and the NextGen models of Allard et al. (1997). The masses of these simulated candidate companions were taken from the radial-velocity data and range from $1-725 M_{\mathrm{J}}$. Ages for the systems were taken from Valenti \& Fischer (2005) and Takeda et al. (2007), which limits the size of our sample but retains a high level of internal consistency.

We split the companions into two groups depending on which model we could use to generate robust magnitudes. The split was made on the basis of companion $T_{\mathrm{EFF}}$ values, with all the companions using the Burrows et al. models

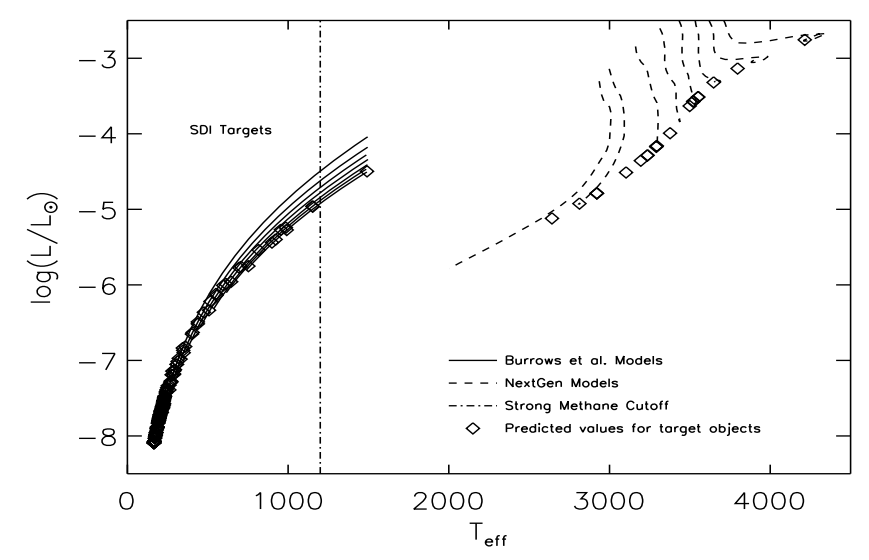

Fig. 1. The solid lines are Burrows et al. (1997) models for different masses and ages. The dashed lines represent a range of masses and ages for the NextGen models of Allard et al. (1997), which are populated by our high-mass targets. The dot-dashed line marks the methane absorption boundary in cool dwarfs and provides a useful upper limit for SDI targets. The discontinuity between the models is the L to T spectral type boundary region, which is not very well modeled, thus the Burrows et al. models were truncated.

having a $T_{\mathrm{EFF}}<2000 \mathrm{~K}$ (which for these older systems corresponds to a $M \sin i<85 M_{\mathrm{J}}$ ) and all other companions using the NextGen models. A custom spline fitting procedure obtained the bolometric luminosities for all the companions (see Fig. 1). The separation in effective temperature between the low temperature models of Burrows et al. and Baraffe et al. and the higher temperature models of Allard et al. is clearly apparent. Taking the whole Butler et al. (2006) catalogue there are 156 companions below the strong methane absorption boundary $(1200 \mathrm{~K})$ (shown by the dot-dashed vertical line on the plot), apart from their low luminosity we expect them to be good SDI targets and a small number of these may well be detectable. There are also 23 companions with $T_{\mathrm{EFF}}$ values above $1200 \mathrm{~K}, 22$ of which have $T_{\text {eff }}>2000 \mathrm{~K}$. These should be observable with conventional AO methods.

The major problem faced when attempting to image close-in companions to bright stars, is the contrast difference. To determine the contrasts between the stars and their companions we had to obtain the JHK magnitudes of the companions, both from internal and reflected flux. To accomplish this the median colors and bolometric corrections (BC) from Leggett et al. (2002) $\left(\mathrm{BC}_{\mathrm{T}}=2.06 ; \mathrm{BC}_{\mathrm{L}}=3.25\right.$, the subscripts denote spectral type $)$ were employed. This correction gave the absolute $K$ magnitudes and, using the colors $\left(H-K_{\mathrm{T}}=-0.04 ; H-K_{\mathrm{L}}=0.70\right)$, we were able to generate their expected absolute $\mathrm{H}$-band magnitudes. For all planetary-mass companions Jupiter characteristics were assumed. We then simplistically simulated the expected reflected flux in the $H$-band and added this component to the internal flux. This was done by using simple geometry and assuming Jupiter's radius and albedo. The total absolute magnitudes of the companions were then subtracted from the absolute magnitudes derived for the primaries giving the estimated contrast $\Delta H$.

Figure 2 shows the contrast and separation estimates from the simulation for all the radial-velocity companions included in this test. The simulated companions represented by filled circles would all have strong methane absorption. The bulk of the objects lie within $0.3^{\prime \prime}$ of the primaries due to the bias introduced by the short radial-velocity baseline. The majority of these lower-mass companions ( $M \sin i<15 M_{\mathrm{J}}$ ) also have high contrasts $(\Delta H \gg 10)$, putting them below the $5 \sigma$ NACO-SDI 


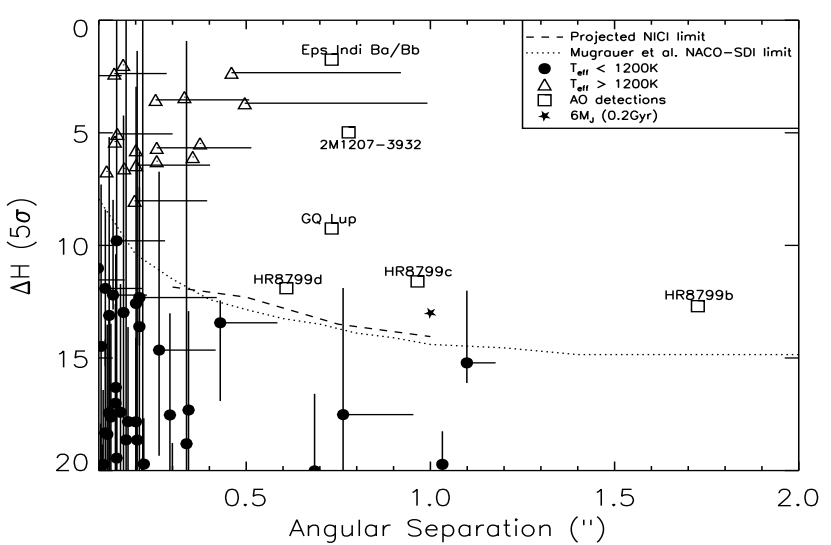

Fig. 2. A subset of our simulated candidate companions, including those imaged in this paper, taken from Butler et al. truncated by $\Delta H$. Triangles represent targets with $T_{\text {eff }}>1200 \mathrm{~K}$, filled circles are targets $<1200 \mathrm{~K}$ (potentially amenable to SDI) and the squares are companions to young stars already found by high contrast AO imaging. For comparison, the dotted line shows the NACO-SDI sensitivity (Mugrauer \& Neuhäuser 2005) and the dashed line shows the NICI sensitivity taken from the Gemini website (http://www.gemini.edu/sciops/instruments/ nici). The error bars represent the range of possible ages from Valenti \& Fischer (2005) and Takeda et al. (2007), along with the error in the $\mathrm{RV}$ measurements. The star represents a $6 M_{\mathrm{J}}$ object at $5 \mathrm{AU}$ orbiting a typical $\mathrm{K} 0$ star with an age of $0.2 \mathrm{Gyr}$ and a distance of $5 \mathrm{pc}$.

threshold. However, two companions have larger separations $>0.3^{\prime \prime}$, approaching the separations of the already discovered objects of Marois et al. (2008), McCaughrean (2003), Chauvin et al. (2004) and Neuhäuser et al. (2005) and, combined with a $\Delta H<13.5$, they could be amenable to SDI imaging. Note that another secure AO detection is Fomalhaut $b$ but this is located far off the plot scale with an angular separation of $\sim 14.9^{\prime \prime}$. The NACO-SDI (Mugrauer \& Neuhäuser 2005) and NICI (http : // www.gemini.edu/sciops/instruments/nici) sensitivities are highlighted in Fig. 2 by dotted and dashed lines respectively. Note that we can not be sure if the Mugrauer \& Neuhauser detectability limits are actual $5 \sigma$ limits or some lower threshold limit. Once the masses and semimajor axes are more precisely defined, the companion magnitudes and separations will most likely increase giving lower contrasts and more viable targets. This has been highlighted on the plot by the error bars which represent the direction in which all companions are expected to move once inclination and eccentricity effects are considered and more RV data points acquired. Another major source of uncertainty is age. For example, a typical $1 \sigma$ age uncertainty for these types of objects is $\sim \pm 2$ Gyr, which translates to a $\sim \pm 2$ mag error in $\Delta H$ with the primary. Due to the high contrast ratios and extremely small separations the majority of these companions are out of reach of current instruments. However, future Extreme-AO systems which are proposing to reach $>15$ mag of contrast may be able to bridge this gap.

All companions with $T_{\mathrm{EFF}}>2000 \mathrm{~K}$ (triangles in Fig. 2 are taken from Nidever et al. 2002 and Jenkins et al. 2010) have $H$-band magnitudes less than 15 , allowing direct imaging using normal AO techniques. Four of these objects have separations larger than $0.35^{\prime \prime}$ and $\Delta H$ less than 8 , making excellent coronographic targets. All planetary-mass companions are off the Fig. 2 plot scale since they have much larger $H$-band contrasts. The star in this figure shows the position of a $6 M_{\mathrm{J}}$ planet in a Jupiter-like orbit as a companion to a $0.2 \mathrm{Gyr}, \mathrm{K} 0$ star at 5 pc. The age and spectral type were chosen since they relate

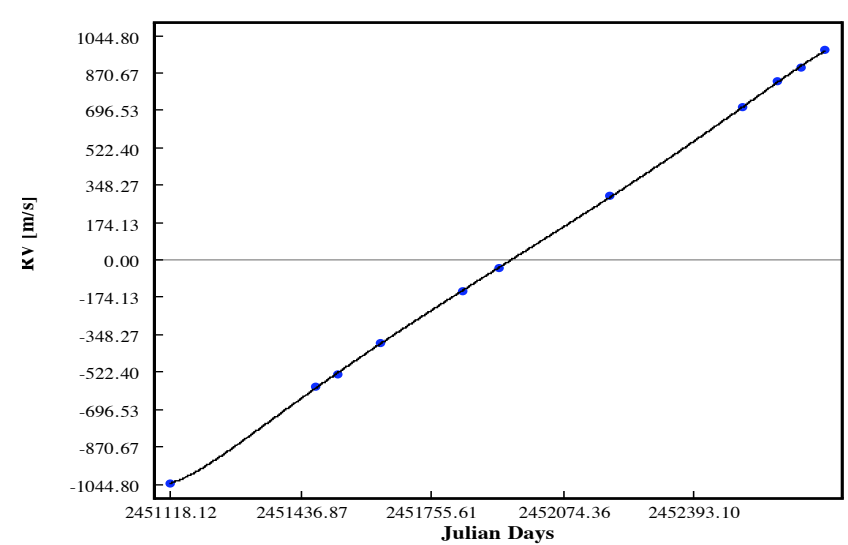

Fig. 3. The radial-velocity measurements for the star HD25874. The minimum-period Keplerian fit to the data yields a period of $\sim 6.5$ years, with a companion minimum mass of $66 M_{\mathrm{J}}$. However, no curvature has been measured therefore the real orbit will be significantly larger than the orbital fit measured here.

to the best case scenario for one of our objects HD120780. It shows that by adopting the lower limit to the large errors on the age of this system that the potential exists to detect planetarymass objects around such stars. Even still, these types of objects reside extremely close to the plotted instrument thresholds, highlighting just how difficult it is to obtain a direct image of any planetary-mass object with the current suite of instruments available. However, radial-velocity studies have revealed a high number ( $\geq 28 \%$ of planet hosting stars) of multiple planet systems (Wright et al. 2009), therefore imaging planet-host stars can provide useful constraints on any longer period, massive companions not yet revealed in the radial-velocity dataset (e.g. Mugrauer et al. 2006; Mugrauer et al. 2007).

\section{Candidate characteristics}

All radial-velocity data in this section were generated using the AAPS and Keck pipelines. These pipelines are still undergoing development following the procedures and techniques described in Marcy \& Butler (1992) and Butler et al. (1996, 2001, 2006). The Keplerian fits shown in Figs. 3-7 are performed using the Systemic algorithm (Meschiari et al. 2009), however we note that most are not very well constrained using the current radialvelocity data. Table 1 lists some relevant information for each object relating to both the radial-velocity and photometric analysis in this work. The parameters and their analysis methods can be found in van Leeuwen \& Fantino (2005), Henry et al. (1996), Valenti \& Fischer (2005), Wright (2005), Jenkins et al. (2006), Takeda et al. (2007) and Jenkins et al. (2008). Tables 2-6 list all radial-velocity data.

\subsection{HD25874}

\subsection{HD120780}

The AAPS has obtained 11 radial-velocity data points over a period of 4.4 years (Fig. 3 data taken from Jenkins et al. 2010). The minimum best-fit Keplerian orbit to this data has an amplitude of $>1000 \mathrm{~m} \mathrm{~s}^{-1}$ relating to a companion period of 6.5 years, eccentricity 0.43 and a minimum mass of $66 M_{\mathrm{J}}$. However, the curvature of the fit has been generated by the algorithm itself as within the uncertainties all the data points lie in a straight line, known as a liner. Therefore, the orbital solutions to this data 


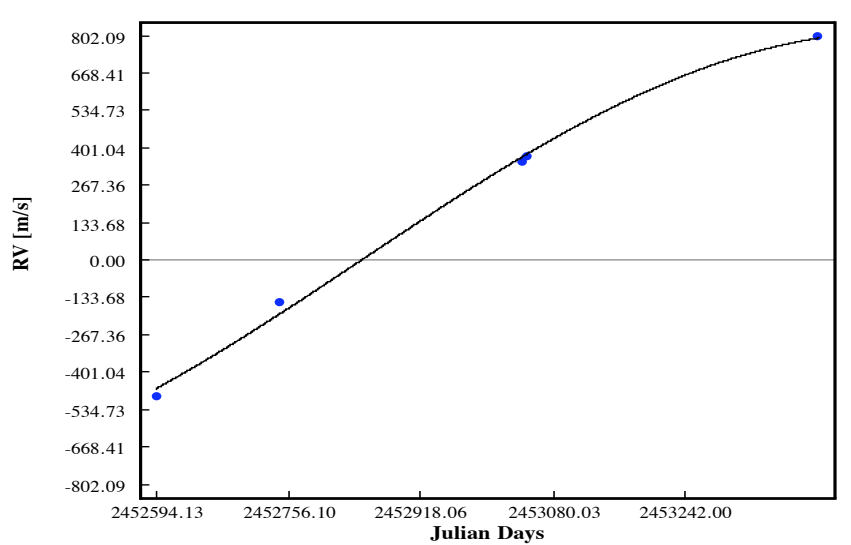

Fig. 4. The best-fit Keplerian orbit to the radial-velocity measurements for the star HD32778, taken by the Keck Planet Search. The fitted orbital period is 6.5 years, with a circular eccentricity and a companion minimum mass of $\sim 55 M_{\mathrm{J}}$. Curvature can be seen in this plot, however with only this limited number of data points and time coverage the fit is still relatively unconstrained.

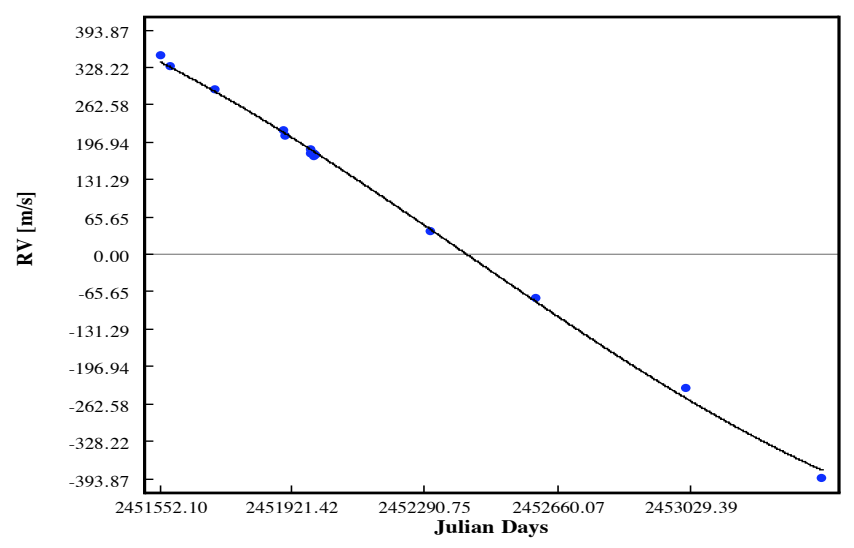

Fig. 5. The orbital solutions for the radial-velocity dataset from the Keck Planet Search for HD91204. All 10 data points are spread across a period of 5 years and the best estimate of the fit has an amplitude of $\sim 1000 \mathrm{~m} \mathrm{~s}^{-1}$ and a period of $\sim 18$ years. This gives a minimum mass for the companion of $\sim 50 M_{\mathrm{J}}$.

series are lower limits. For comparison the best-fit Keplerian with twice the orbital period would relate to a companion minimum mass of $\sim 190 M_{\mathrm{J}}$ and similar $\chi^{2}$ of 2.5 . From experience we estimate the lower limit of the period of the orbit to be around four times larger than currently estimated. If we take the period range 6.4-25.8 years and the Hipparcos distance of $25.91 \mathrm{pc}$, the projected separation will be in the range $0.13-0.34^{\prime \prime}$. The absolute $H$-band magnitude is 3.20 mag and our estimation for the absolute $H$-band magnitude of the companion using the technique in Sect. 3.1 is $15.31 \mathrm{mag}$, giving a best estimate for the contrast ratio upper limit of $12.11 \mathrm{mag}$.

\section{3. $H D 32778$}

Five radial-velocities over a period of 2.25 years for this object (Fig. 4) and the best-fit Keplerian orbit has a semi-amplitude of $\sim 750 \mathrm{~m} \mathrm{~s}^{-1}$. This is consistent with a companion with a minimum mass of $\sim 55 M_{\mathrm{J}}$, a period of 6.5 years and a circular eccentricity. Even though this is not a liner the limited amount of data points and temporal coverage means this is not very well constrained. The small amount of curvature does help better

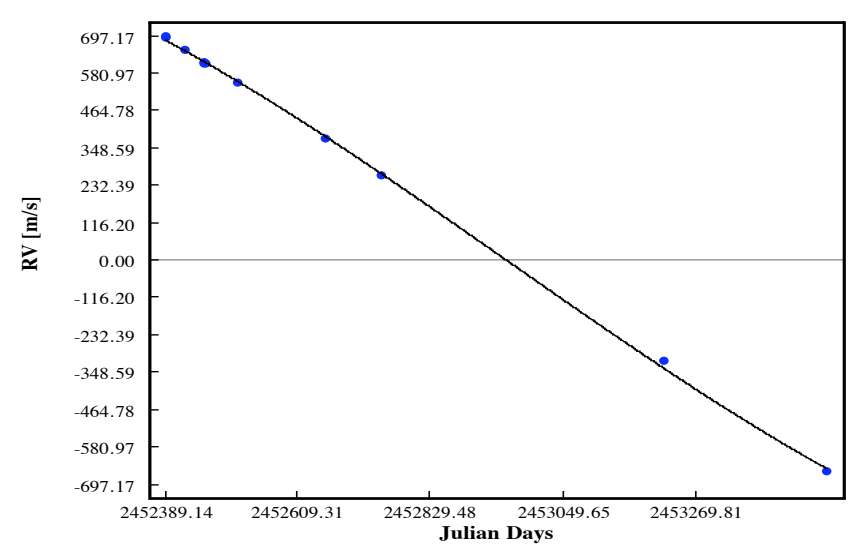

Fig. 6. The radial-velocity dataset and fits for the companion to the star HD120780. The minimum-period solution to the large trend is a companion with a period of $\sim 12$ years and minimum mass of $\sim 70 \mathrm{M}_{\mathrm{J}}$, placing it towards the upper end of the brown dwarf regime. However, this is a liner and the actual mass of the companion is probably significantly higher than this.

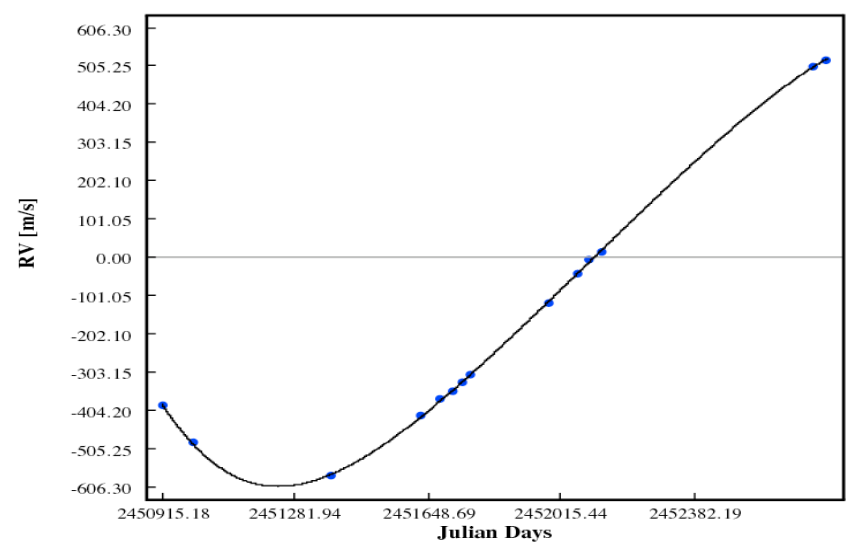

Fig. 7. The radial-velocity dataset from the AAPS for the star HD145825. The best-fit Keplerian orbit to the 14 data points are shown, along with the estimated orbital solutions. Curvature can clearly be seen in this data, and since this is not a liner the orbital solution is better constrained than all the other four stars. The measured semi-amplitude of $617 \mathrm{~m} \mathrm{~s}^{-1}$ indicates the presence of a companion with a period of 7.8 years, and eccentricity of 0.2 and a minimum mass of $44.5 M_{\mathrm{J}}$.

constrain the orbit since if we look at the best-fit for twice the orbital period we quote here, we find a minimum mass of $137 M_{\mathrm{J}}$ but with a very high $\chi^{2}$ of 186 , showing such large orbits are difficult to fit well. At a distance of $22.48 \mathrm{pc}$ the estimated angular separation for this companion is $0.16^{\prime \prime}$. The absolute $H$-band magnitude of this star is 3.71 and with an estimated maximum absolute $H$ of 15.59 using the current fit and the lower age limit, the contrast would be $\sim 12$ mag.

\section{4. $H D 91204$}

Figure 5 shows the orbital fit to the large trend in the radialvelocity dataset and again it appears to be a liner. The estimate to this data has an amplitude of $1000 \mathrm{~m} \mathrm{~s}^{-1}$, a period of $\sim 18$ years and circular eccentricity. The minimum mass of the companion to this fit is $\sim 50 M_{\mathrm{J}}$ but yet again this is a liner fit to the data and therefore the expected orbital period will be underestimated. Again, twice the orbital period would give rise to a companion 
Table 1. Parameters for all stars in this work.

\begin{tabular}{ccccccccc}
\hline \hline Star & $V(\mathrm{mag})$ & $d(\mathrm{pc})$ & $T_{\mathrm{EFF}}(\mathrm{K})$ & Mass $\left(M_{\odot}\right)$ & Radius $\left(R_{\odot}\right)$ & Age $(\mathrm{Gyr})$ & {$[\mathrm{Fe} / \mathrm{H}]$} & $\log R_{\mathrm{HK}}^{\prime}$ \\
\hline HD25874 & 6.74 & 25.91 & 5699 & $1.14 \pm 0.09$ & $1.10 \pm 0.03$ & 9.36 & -0.02 & -4.95 \\
HD32778 & 7.02 & 22.48 & 5652 & $0.95 \pm 0.08$ & $0.86 \pm 0.01$ & 10.30 & -0.48 & -4.87 \\
HD91204 & 7.82 & 51.55 & 5914 & $1.05 \pm 0.18$ & $1.22 \pm 0.06$ & 5.16 & +0.17 & -5.09 \\
HD120780 & 7.37 & 17.01 & 5008 & $0.60 \pm 0.05$ & $0.70 \pm 0.01$ & 5.40 & -0.26 & -4.79 \\
HD145825 & 6.55 & 21.55 & 5803 & $1.08 \pm 0.10$ & $0.97 \pm 0.02$ & 1.92 & +0.03 & -4.74 \\
\hline
\end{tabular}

Table 2. HD25874 radial-velocity data.

\begin{tabular}{ccc}
\hline \hline $\mathrm{JD}$ & $R V(\mathrm{~m} / \mathrm{s})$ & $\sigma_{\mathrm{rv}}(\mathrm{m} / \mathrm{s})$ \\
\hline & & \\
2451118.122 & -904.1 & 3.4 \\
2451473.261 & -453.1 & 4.5 \\
2451526.013 & -394.2 & 3.4 \\
2451630.876 & -252.7 & 3.4 \\
2451830.118 & -5.9 & 4.3 \\
2451920.038 & 99.6 & 4.3 \\
2452189.177 & 436.6 & 5.1 \\
2452511.239 & 850.3 & 8.4 \\
2452594.081 & 968.5 & 4.6 \\
2452654.062 & 1032.4 & 4.1 \\
2452710.892 & 1113.6 & 3.2 \\
\hline
\end{tabular}

Table 3. HD32778 radial-velocity data.

\begin{tabular}{ccc}
\hline \hline JD & $R V(\mathrm{~m} / \mathrm{s})$ & $\sigma_{\mathrm{rv}}(\mathrm{m} / \mathrm{s})$ \\
\hline & & \\
2452594.134 & -841.5 & 2.0 \\
2452744.876 & -502.5 & 2.1 \\
2453042.025 & 0.0 & 1.8 \\
2453046.980 & 19.8 & 2.8 \\
2453402.991 & 448.1 & 2.0 \\
\hline
\end{tabular}

Table 4. HD91204 radial-velocity data.

\begin{tabular}{ccc}
\hline \hline $\mathrm{JD}$ & $R V(\mathrm{~m} / \mathrm{s})$ & $\sigma_{\mathrm{rv}}(\mathrm{m} / \mathrm{s})$ \\
\hline 2451552.102 & 172.2 & 1.5 \\
2451581.998 & 152.6 & 1.4 \\
2451706.816 & 112.0 & 1.5 \\
2451898.177 & 38.8 & 1.5 \\
2451901.157 & 29.2 & 1.5 \\
2451972.066 & 0.0 & 1.4 \\
2451973.023 & 5.4 & 1.4 \\
2451981.986 & -4.8 & 1.3 \\
2451982.973 & -1.0 & 1.3 \\
2451983.978 & -4.3 & 1.4 \\
2452307.969 & -137.3 & 1.6 \\
2452601.140 & -254.2 & 1.5 \\
2453017.162 & -411.7 & 1.5 \\
2453397.979 & -570.6 & 1.4 \\
\hline
\end{tabular}

with a minimum mass of $110 M_{\mathrm{J}}$ but with a fairly high $\chi^{2}$ of 15 . From the estimated orbital period above of $\sim 18$ years, and the distance to the star of $51.55 \mathrm{pc}$, we expect the lower limit on the separation to be $\sim 0.13^{\prime \prime}$. The absolute $H$-band magnitude for HD91204 is 2.83 mag with the estimated absolute magnitude for the companion of $\sim 16.05 \mathrm{mag}$, relating to an upper limit for the contrast ratio of $13.22 \mathrm{mag}$ at the given age of the system.

Eight data points have been acquired over a period of $\sim 3$ years and a linear fit has been plotted through the data (Fig. 6). The best minimum estimate to the orbital solution gives an orbital period for the companion of $\sim 12$ years and assuming a
Table 5. HD120780 radial-velocity data.

\begin{tabular}{ccc}
\hline \hline $\mathrm{JD}$ & $R V(\mathrm{~m} / \mathrm{s})$ & $\sigma_{\mathrm{rv}}(\mathrm{m} / \mathrm{s})$ \\
\hline 2452389.145 & 85.9 & 1.4 \\
2452390.076 & 79.7 & 1.3 \\
2452422.026 & 42.3 & 1.4 \\
2452452.991 & 3.8 & 1.1 \\
2452454.920 & -0.3 & 1.0 \\
2452455.936 & -0.1 & 1.1 \\
2452509.881 & -59.6 & 1.7 \\
2452655.135 & -230.7 & 2.8 \\
2452748.038 & -345.9 & 1.4 \\
2453217.880 & -923.7 & 1.9 \\
2453489.100 & -1268.8 & 1.5 \\
\hline
\end{tabular}

Table 6. HD145825 radial-velocity data.

\begin{tabular}{ccc}
\hline \hline $\mathrm{JD}$ & $R V(\mathrm{~m} / \mathrm{s})$ & $\sigma_{\mathrm{rv}}(\mathrm{m} / \mathrm{s})$ \\
\hline 2450915.182 & -222.3 & 2.2 \\
2451002.046 & -322.3 & 3.0 \\
2451382.974 & -408.3 & 1.9 \\
2451630.280 & -250.1 & 2.0 \\
2451683.047 & -204.4 & 2.1 \\
2451718.096 & -185.1 & 2.2 \\
2451742.997 & -162.1 & 2.1 \\
2451766.897 & -143.4 & 1.8 \\
2451984.224 & 46.2 & 2.3 \\
2452060.982 & 125.7 & 2.0 \\
2452091.945 & 160.8 & 2.1 \\
2452126.927 & 182.4 & 2.4 \\
2452711.315 & 673.1 & 3.1 \\
2452748.215 & 690.2 & 3.5 \\
\hline
\end{tabular}

circular orbit, a minimum mass of $\sim 70 M_{\mathrm{J}}$. In this case the comparison minimum mass for twice the orbital period is $145 M_{\mathrm{J}}$, with a $\chi^{2}$ of 18 , much lower than the best minimum estimate. In fact, searching the parameter space freely for the best single companion solution to this data returns a companion with a minimum mass of $1.1 M_{\odot}$ and orbital period of over 56 years. Clearly such a companion would manifest itself in the stellar spectra and this is not the case, which could indicate the need for a double companion solution for this star. However, taking the 12 year period we get a semimajor axis of $\sim 5.24 \mathrm{AU}$ and at a distance of $17.01 \mathrm{pc}$, the angular separation would be $\sim 0.31^{\prime \prime}$. The absolute $H$-band magnitude of the star is 4.3 mag and with an estimated lower limit to the absolute $\mathrm{H}$ for the companion of $\sim 9.88 \mathrm{mag}$, the estimated upper limit to the contrast ratio is $5.58 \mathrm{mag}$. This represents the lowest contrast estimate for the five objects and arises due to the extremely small lower age estimate of $0.2 \mathrm{Gyr}$ from Valenti \& Fischer (2005). Note however that this age is unconstrained as the upper age estimate reaches as high as the age of the universe i.e. $13.4 \mathrm{Gyr}$. 


\subsection{HD145825}

Figure 7 shows the 14 radial-velocity measurements made by the AAPS over a period of $\sim 5$ years. This allows a constrained fit to the points as the fitting algorithm has one inflection and significant secondary inflection curvature to describe. The best-fit Keplerian orbit finds solutions that best reproduce the observed curve, with a semi-amplitude of $617 \mathrm{~m} \mathrm{~s}^{-1}$, described by a companion with an orbital period of 7.8 years, an eccentricity of 0.2 and a minimum mass of $44.5 M_{\mathrm{J}}$. Since a significant percentage of the orbit has been observed no comparison fit is necessary as this fit describes the data very well. The semimajor axis of this orbit is $4.07 \mathrm{AU}$ and at a distance of $21.55 \mathrm{pc}$ the apparent separation of the companion would be $\sim 0.19^{\prime \prime}$. However, even though the solutions are better constrained than the liner plots, they are still lower limits as only one inflection is securely found and it is likely that, at best, only $2 / 3 \mathrm{rd}^{\prime}$ s of the orbit has been mapped. The star's absolute H magnitude is 3.39 mag and with the best estimates of the companion's absolute $\mathrm{H}$ set at $14.2 \mathrm{mag}$, the upper limit on the contrast ratio is estimated to be $10.8 \mathrm{mag}$.

\section{NACO imaging}

\subsection{Observations and reduction}

The observations of each of the stars chosen as primary candidates were carried out on 02 March 2006 using the NACOSDI instrument mounted on the 8m ESO VLT4-Yepun telescope in Paranal, Chile. The average seeing throughout the observing night was $\sim 0.8^{\prime \prime}$. The NACO AO system is described in detail in Rousset et al. (2003). Since all targets in this project are very bright $(V<8)$ the star itself was chosen as the guide.

The SDI system employs a double calcite Wollaston prism to split the incoming beam into four separate beams and then feeds them through a quad $\mathrm{CH} 4$ filter that is set in the focal plane. The filters are set at central wavelengths of $1.575 \mu \mathrm{m}$ (F1), $1.600 \mu \mathrm{m}(\mathrm{F} 2)$ and $1.625 \mu \mathrm{m}$ (F3a and F3b) and with bandpasses of $0.025 \mu \mathrm{m}$, which helps to limit residuals due to speckles and calcite chromatics. The differential non-common path errors are less than $10 \mathrm{~nm}$ rms per Zernicke mode between the beams (Lenzen et al. 2004). In this configuration the telescope has a projected field of view of 5 arcsec square, reduced to $2.7 \times 3.7$ arcsec after the tilt of $133^{\circ}$ from the SDI focal plane mask during this run is considered, and has a camera plate scale of $0.017^{\prime \prime}$ pixel $^{-1}$.

The observations were performed using an 8-point jitter pattern. One of the jitter frames was a pure sky-frame to better aid in sky background removal. Each jitter integration (Detector Integration Time aka DIT) ranged from 2-6 s depending on the brightness of the central PSF. Each DIT was determined by increasing the time until the central few pixels of the star were saturated, allowing very high $\mathrm{S} / \mathrm{N}$ in the halo of the PSF. However, we believe that we could further increase this $\mathrm{S} / \mathrm{N}$ by increasing the DIT's and saturating more of the stellar PSF, since we will gain a higher dynamic range. Each observation took around $60 \mathrm{~min}$ to complete, consisting of 44 jitter cycles per star.

The reduction of all the NACO-SDI raw data made use of the custom pipeline of Biller et al. (2004, 2007). First, all the raw frames were cleaned for any background sky noise by subtracting out the sky image from the jitter cycle. A standard flat-field is then applied by combining the flat images into a master flat and dividing out the pixel-to-pixel variations from each individual image frame. To further clean the image a bad pixel map is created from the jittered images to flag any dead pixels on the CCD

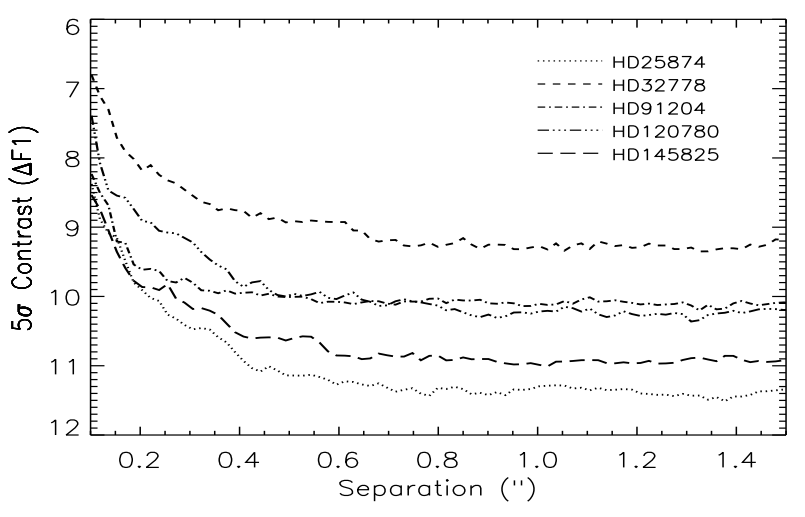

Fig. 8. A combined plot showing all the contrast curves $(\Delta \mathrm{F} 1)$ for each star plotted on a similar scale. The stars and their associated curves are indicated. It is clear that there is a large spread in contrasts, with both HD25874 and HD145825 exhibiting similar contrast depths which are $>2$ mag deeper than the shallowest curve, HD32778. Note however that both HD32778 and HD120780 had their contrasts estimated from the other stellar data due to their saturated acquisition frames.

chip and these are removed from all image frames. Apertures are then extracted around each filtered image and the Airy pattern and flux is scaled. The frames are then unsharp masked by dividing through by a heavily smoothed version of the original image. A shift and subtract algorithm is used to align the jittered images, with the first frame in the series used as the reference image and all other images aligned to this first image.

\section{Data analysis}

Once all data frames have been fully reduced the next step is to setup the analysis procedure. This was done by adding and subtracting the various narrow-band filters across the methane feature to provide the best conditions for detecting the faint companions. The two combinations that provide the best contrasts and $\mathrm{S} / \mathrm{N}$ ratios to detect cool dwarfs target both $\mathrm{M}$, L-type and $\mathrm{T}$, Y-type objects. The SDI instrument, by its pure design, is built to search for companions of mid-T spectral type or later, since the subtraction across the methane band suppresses the starlight and speckle pattern to highlight objects with strong methane absorption in their atmospheres. However it can also be used to search for L-dwarfs by combining all the filters to create a broadband image that would detect any L-dwarf signature. This, however, is heavily limited by the bright star and the bright super-speckles in the image. We employed both these approaches to search for faint companions around the five stars in this project.

Before discussing each system individually, Fig. 8 shows the SDI reduced contrasts for all the systems on the same plot and scale. The key in the upper right of the plot indicates which curve represents which system. Since the SDI reduced curve represents the limiting $5 \sigma$ contrast for each system this plot highlights how deep the observations reach over the parameter space sampled. The contrast curves were estimated by defining a $5 \times 5$ square pixel box, placing the box at the center of the images and then calculating the standard deviation within the box whilst moving it outwards from the center, pixel-by-pixel, in 8 different directions, separated by $45^{\circ}$ angles. Averaging these gives a measure of the sky background noise. To measure the contrast ratio, these counts were divided by the peak flux estimate for each star. Peak flux was estimated from the unsaturated acquisition image, scaling to the appropriate exposure time for the saturated data images. For two of these curves however, representing the 


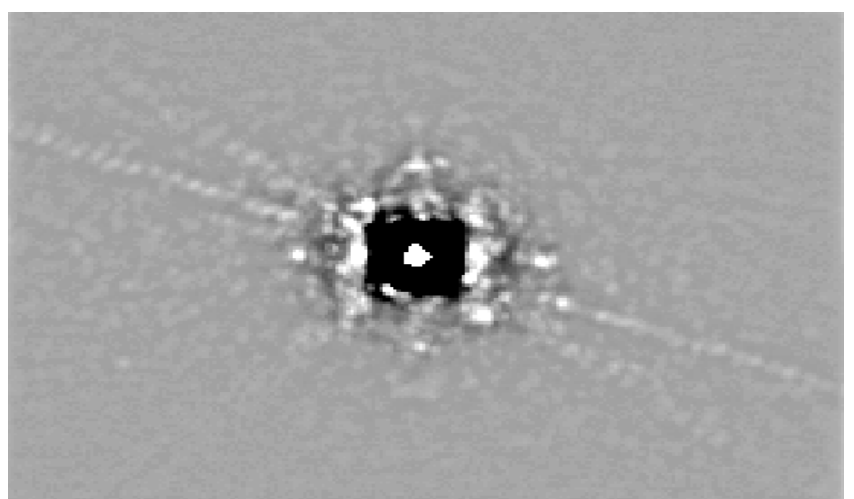

Fig. 9. A broadband image of the star HD25874. The image was constructed by combining all images through the three separate filter bandpasses. Here a number of super-speckles can clearly be seen scattered across the image, the brightest of them reside close to the central star. All the possible cool dwarf companions visible in this image are ruled out as speckles when comparing the rotated images.

stars HD32778 (short dashed line) and HD120780 (three dotsingle dashed line), there are no unsaturated acquisition images and hence their flux counts were estimated using the data from the other stars with unsaturated images. The 2MASS $H$ magnitude for each was plotted against peak flux in the acquisition images. A polynomial was then fit to this data and by taking the $H$ magnitude for the saturated stars we could calculate their expected peak counts. This returns an estimate and was only employed since the observational methodology was similar. There is a large spread in contrast limit in this data with a difference of $\leq 2.2$ mag between HD32778 and HD25874 (dotted line), with HD32778 being the least sensitive (max contrast of $9.3 \mathrm{mag}$ ) and HD25874 the most sensitive systems (max contrast of $11.5 \mathrm{mag}$ ). For all systems the curves are flat beyond $0.7^{\prime \prime}$ and remain so out to the edges of the scale. No additional companions with larger separations than those indicated in the radial-velocity datasets were found in any of these systems.

\subsection{M,L-Dwarfs}

As mentioned above to extract the signal from companions that don't exhibit strong methane in their atmospheres the best SDI approach is to combine all the narrow-band filters employed by the SDI device and create a broadband image that will increase the S/N of any cool companion. This method was applied to the data frames from all five of the target stars. Note that a targeted search for such objects that don't exhibit strong methane absorption in their atmospheres would benefit significantly from utilising a standard broadband $\mathrm{AO}$ approach, since with the narrowband $S D I$ filters there is a loss of efficiency and hence a loss of contrast for a given total integration time. To help with the detection of any signals and to decipher them from residual stellar speckles all images were taken at two different rotation angles, separated by $33^{\circ}$ on the sky. By blinking these rotated broadband images it is possible to spot any detection that appears at different positions in the rotated images. Any super-speckles should remain in the same place throughout the roll angle, therefore a real detection can be picked out in a field with a number of residual speckles. Figure 9 is an example of one of these broadband images for the star HD25874.

From the estimates of the companion minimum masses generated from the orbital fits we might expect three of these companions to reside in the L-dwarf regime and considering the limited data they may also have M dwarf masses. However the estimates of the contrast ratios are high and as the contrast that can be reached by using a broadband image is significantly lower than the subtracted images, it would prove extremely difficult to detect any of these L-dwarfs using this method. Indeed, the search employed for L-dwarfs around all five of these stars turned up a number of bright objects, these can be seen as the spots spread across the image in Fig. 9. However, none of these detections fulfilled the rotation roll angle criteria, indicating they are residual speckles. It is clear that without looking for roll angle modulation it is extremely difficult to differentiate between a speckle and a real object.

If the companions to these stars are L-type objects and the estimated separations from the radial-velocity curves are 0.13" (HD25874), 0.16" (HD32778), 0.13" (HD91204), 0.31" (HD120780) and 0.19" (HD145825) then they must not have contrasts less than $6.7,7.4,7.0,7.9$ and 7.8 mag respectively (taken from the conventional AO (dot-dashed) curves in Figs. 10, 13 and 15). Note that the right-hand $y$-axes in these figures show the estimated masses at the age of these systems relating to contrast limits for methane objects only and this will be explained in Sect. 5.2. At the estimated separations the mass limits for L-dwarfs are 87, 77, 92, 72 and 72 Jupiter-masses respectively. No L-type objects with the given ages and separations can have masses above these limits. Also shown in these plots represented by the solid curves are the radial-velocity sensitivities. These confidence limits from the radial-velocity data sets are explained below and the conclusions drawn are presented there.

\subsection{T or Y-Dwarfs and Exoplanets}

The search for T and Y-dwarfs, along with planetary mass objects, cooler than $1200 \mathrm{~K}$ is accomplished by subtracting the images inside and outside of the methane band at $1.62 \mu \mathrm{m}$. The filters employed for this task are the $F 1$ filter centered at $1.575 \mu \mathrm{m}$ and the $F 3 a$ filter centered at $1.625 \mu \mathrm{m}$ (Close et al. 2005). This subtraction, which we assign $\Delta \mathrm{F} 1$, helps to suppress the Airy rings from the stellar PSF, and attenuates the speckle pattern that is a function of the stellar beam. As there is no strong absorption at $1.62 \mu \mathrm{m}$ in the stellar atmosphere, the subtraction removes the stellar light leaving behind the faint methane signature from the cool companion. Similar to the search for M, L-type objects the $33^{\circ}$ roll angle allows one to distinguish between a genuine cool companion signal and any residual speckles.

For each of the five stars we performed this search on, two candidates fulfilled the roll angle criteria, found around the stars HD25874 and HD120780, both of which are discussed in the next section. For the three other stars no significant companions are seen in the subtracted images across the roll angle. The SDI reduced contrast limits in Fig. 10 (dashed line) for the stars HD32778, HD91204 and HD145825 tell us that if the radialvelocity companions that were targeted were T or Y-dwarfs, they can not have absolute $H$ magnitudes $<\sim 11.1,11.5$ and 13.2 mag respectively, at the estimated separations for these objects. All $H, M_{\mathrm{H}}$ and mass estimates are assuming T5-type status, including the right-hand $y$-axes, which show the expected masses for the corresponding contrast ratios at the estimated age of the star. We chose a T5 simply because it represents the middle of the T-dwarf regime and since these objects are expected to be old we might expect them to reside somewhere close to this spectral type. A reference such as a $\mathrm{T}$ dwarf was necessary due to the unique nature of the SDI observations, since they utilize 

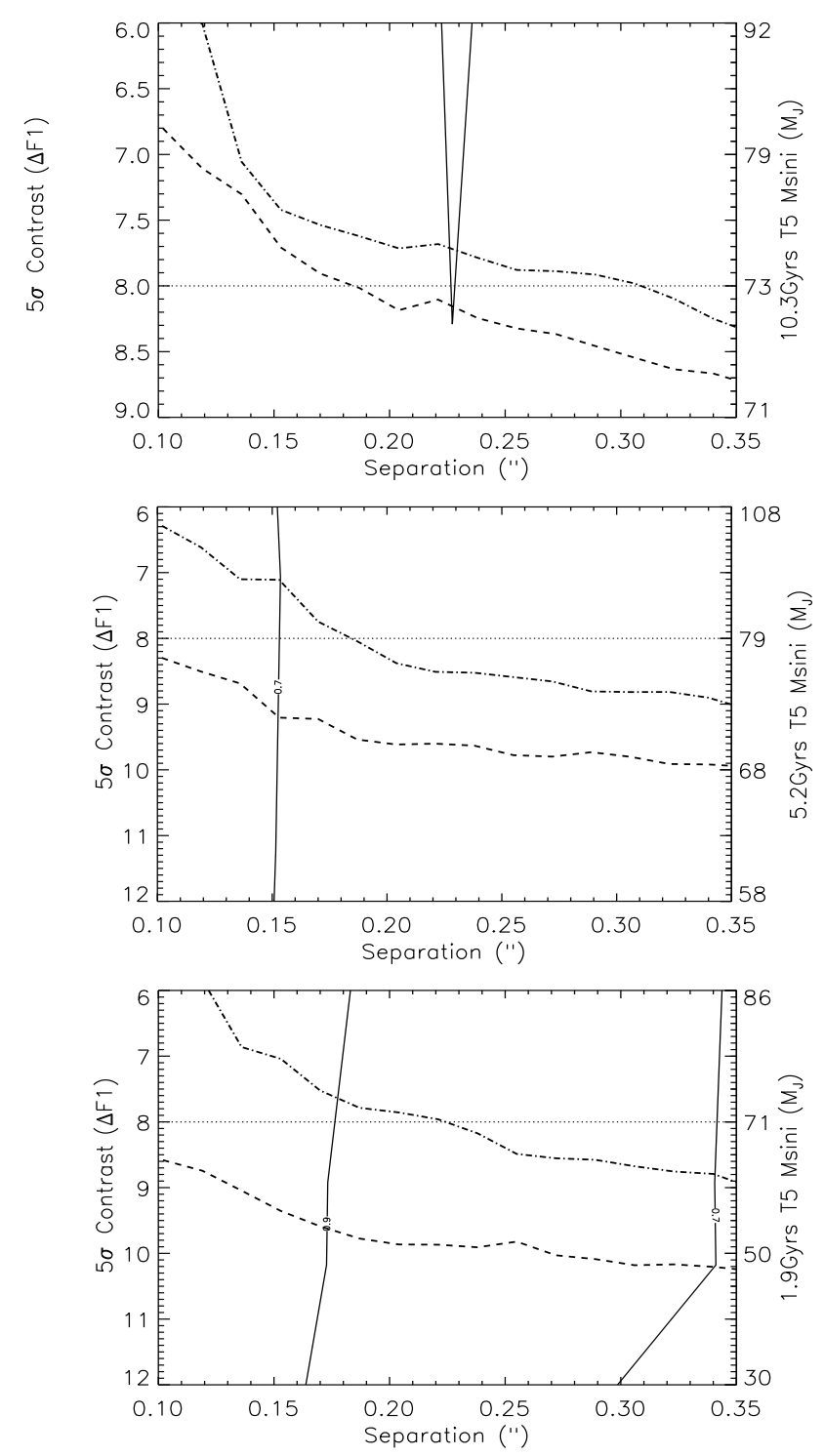

Fig. 10. The $5 \sigma$ contrast limits $(\Delta \mathrm{F} 1)$ for the stars HD32778 (top panel), HD91204 (middle panel) and HD145825 (bottom panel). The dot-dashed curves represent the Conventional AO detection thresholds and the dashed curves represent the SDI reduced thresholds. No detections were found around any of these stars. The solid curves represent the confidence limits we are sensitive too from the radial-velocity data sets. Both HD32778 and HD91204 cover only a limited parameter space given by the radial-velocity data, which means the companion is still relatively unconstrained. Whereas, HD145825 observations reach deeper and are able to rule out a lot of solutions for the companion. The right-hand axes show companion masses for a T5 dwarf at the best estimated age of the systems from Valenti \& Fischer (2005) and Takeda et al. (2007) and are for comparison only. The horizontal dotted line represents the approximate strong methane boundary.

narrow-band filters across a strong absorption feature and objects with more or less methane absorption with have more or less flux entering the $F 3 a$ filter. Since T5 objects possess a strong methane break they provide good references for comparison in our contrast curves. A correction must be made to compare with model absolute $H$-band magnitudes and we simply apply an offset of +0.5 mag for a typical T5 dwarf (Biller et al. 2007).
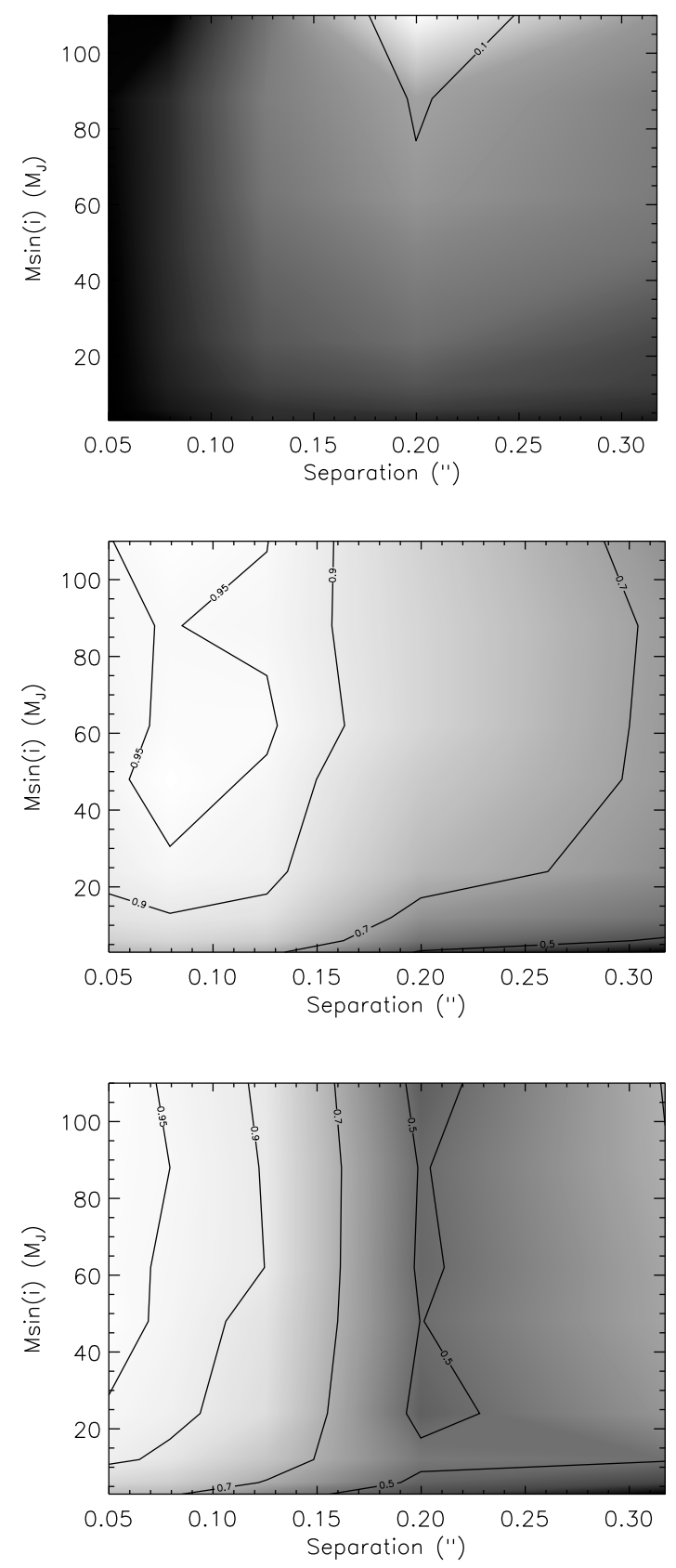

Fig. 11. The doppler sensitivity confidence limits in mass-angular separation space for the stars HD32778 (top panel), HD91204 (middle panel) and HD145825 (bottom panel). These confidence limits have been integrated over all eccentricities and the percentage limit is shown inside each curve. The gray scale highlights the changing spacial confidence limits with dark the least constrained through to the most constrained regions being lightest. Only HD145825 has fairly well constrained doppler data out to reasonable NACO-SDI sensitive separations. Whereas for HD32778 there is no real constrained parameters below the hydrogen burning limit.

\subsection{Doppler sensitivities}

We have performed a sensitivity analysis of each individual system to determine the confidence limits from the radialvelocity datasets and how these limits transfer into the contrast curve parameter space. The sensitivities were determined using the method explained in O'Toole et al. (2009) with a small modification. In brief, simulations of radial-velocity curves are 
analysed using a two-dimensional Lomb-Scargle periodogram. The simulations covered the mass range $3-110 M_{\mathrm{J}}$, period range $300-4800 \mathrm{~d}$ and eccentricities from 0.0 to 0.8 in steps of 0.1 . Jitter values for each star, based on the method of Wright (2005), were incorporated into the simulations. The resulting fits were checked against five detection criteria: the four described in O'Toole et al. and a fifth based on the rms of the data, described here. If the measured period is $50 \%$ longer than the time baseline of the dataset, a linear fit to the dataset is performed. This fit is then subtracted from the data and the rms of the residuals are compared to the rms before the linear trend was subtracted. A detection is made if the rms drops by a factor two or more throughout this process. When used in conjunction with the SDI contrast curves, these doppler sensitivies can help to more accurately constrain the parameter space that we have covered and help us to determine what possible companion objects the data did not detect. Sensitivities are shown as solid curves in Figs. 10 and 11 .

The radial-velocity confidence limits for HD32778 (Fig. 10 top) show that due to the lack of data points for this star we have no strong constaint on any sub-stellar companions. The curve here represents the $10 \%$ confidence limit for this star (shown more clearly in the top plot of Fig. 11) and therefore below the hydrogen burning limit we have no real constraints on any companions in this parameter space. The gray scale in this figure, and the following detectability curves, show the changing confidence limits running from dark being the least constrained to light the most constrained regions. Given the lack of data here there is only a very small region where we can rule out any companions with an confidence and most of the parameter space is highly unconstrained. The $10 \%$ confidence bound barely reaches beyond the $5 \sigma$ ( $99.9 \%$ confidence limit) of the SDI reduced contrast curve and only covers a very small part of the parameter space beyond the AO curve. Hence, for this system the radialvelocity data does not help to constrain the companion and any information we can extract about the unseen companion is drawn from the contrast curves alone. This leads to the probability that the companion is of lower mass $\left(\sim \leq 70 \mathrm{M}_{\mathrm{J}}\right)$ with a fairly unconstrained orbital separation i.e $>0.076^{\prime \prime}$ from the baseline of the radial-velocity data.

Similar to HD32778, the confidence limits in massseparation space for HD91204 are poorly constrained (Fig. 10 middle). However, since HD91204 has a larger database of velocities than HD32778, we can say to a $70 \%$ confidence level that the companion to this star is not a close by $\left(\leq 0.15^{\prime \prime}\right)$ substellar or stellar secondary. The middle panel in Fig. 11 shows the mass-separation parameter space for this star, reaching down onto the stars surface. Clearly very close-by companions can be ruled out to high levels of confidence due to the larger number of data points and the fact that this radial-velocity curve is a liner. This is further highlighted by the large light region shown in the gray scale and a lack of any large contrast gradient. Objects with separations below $0.06^{\prime \prime}$ and masses above around $30 M_{\mathrm{J}}$ can be ruled out to $\sim 90-95 \%$ confidence and moving out to separations of $0.08^{\prime \prime}$ we can still rule out objects down to around the planetary mass limit at the $90 \%$ level of confidence. Therefore, we can say that the companion to this star likely has a fairly large separation and is a faint substellar companion or, as for almost all of the imaged objects, the companion has a longer orbital period, but the inclination of the system was such that when we were observing the companion it was hidden behind the star at on-sky angular separations below $0.1^{\prime \prime}$.

In comparison to these other two stars, HD145825 has enough data points and exhibits enough curvature in the
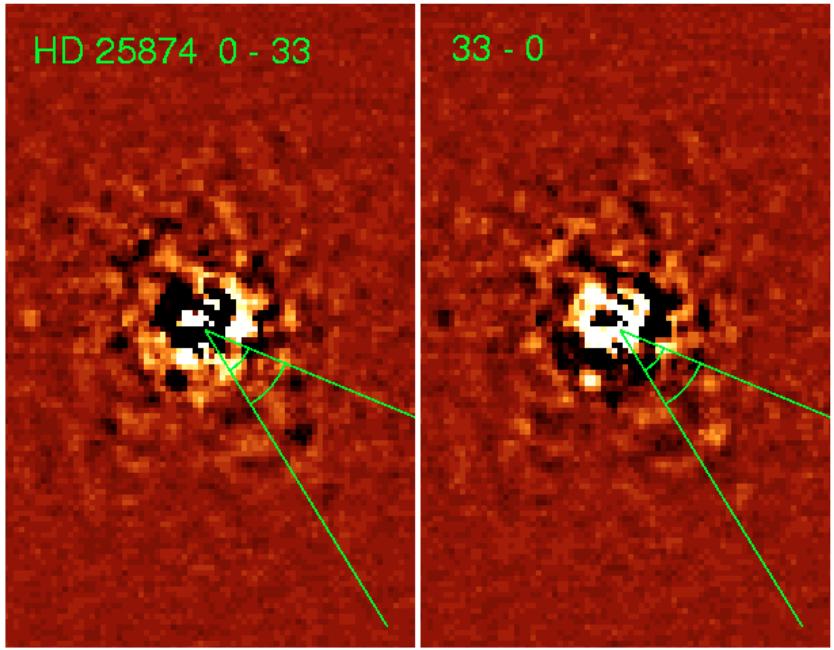

Fig. 12. An annotated image of the star HD25874 at both inverted roll angles. The lines mark the $33^{\circ}$ projection of rotation of the camera. The negative and positive detections of the possible source companion intersects both these lines at the projected separation of $\sim 0.29^{\prime \prime}$.

timeseries that fairly high levels of confidence from the velocities overlap with the $99.9 \%$ confidence limits from the imaging work. The bottom panel in Fig. 10 shows that below the SDI curve we still have over $90 \%$ confidence in ruling out close by $\left(\leq 0.17^{\prime \prime}\right)$ objects down to low brown dwarf masses. In addition, the $95 \%(2 \sigma)$ confidence limit can rule out a lot of possible brown dwarf/stellar companions below the $0.1^{\prime \prime}$ angular separation limit of the SDI technique (Fig. 11 bottom). The gray scale reveals more structure than HD32778 and HD91204 due to the significant curvature in the velocities. Particularly we can see that the region around $0.2^{\prime \prime}$ separation is less constrained than inside and outside this separation and due to the indication of secondary curvature in the velocities, we arrive at fairly high confidence levels beyond $0.3^{\prime \prime}$ separation.

From these combined constraints we can rule out to really high levels of confidence any brown dwarf/stellar companions with small separations (short period orbits). Also, at the $1 \sigma$ level we can say that there are no objects at all below a separation of around 0.34 " with masses above $40 M_{\mathrm{J}}$ and also no companions down into the giant exoplanet regime within $0.20^{\prime \prime}$. These combined data sets argue for the companion to HD145825 to be an extremely faint sub-stellar companion with a moderate separation.

\section{HD25874 and HD120780 detections?}

Out of the five stars that we searched around, two possible detections were made around the stars HD25874 and HD120780. Both of these candidates fulfilled the requirements to be considered as bona fide candidates as they were bright sources, that had counterparts at $33^{\circ}$ in the rolled images. However, after careful analysis we believe these to be artifacts of the reduction procedure and not true companion objects. Both will be discussed here.

Figure 12 shows the $33^{\circ}$ roll angle of the camera and how it projects along the image through the $T 3$ filter $(\mathrm{F} 1(1.575 \mu \mathrm{m})$ $\mathrm{F} 3 \mathrm{a}(1.625 \mu \mathrm{m}))$ for HD25874. The detections are found at the ends of the second arc along the projection with a separation from the central pixel of $0.29 \pm 0.01^{\prime \prime}$ and a position angle of $240^{\circ}$. This enhanced image highlights more of the speckles across the images in both negative and positive formats e.g. 

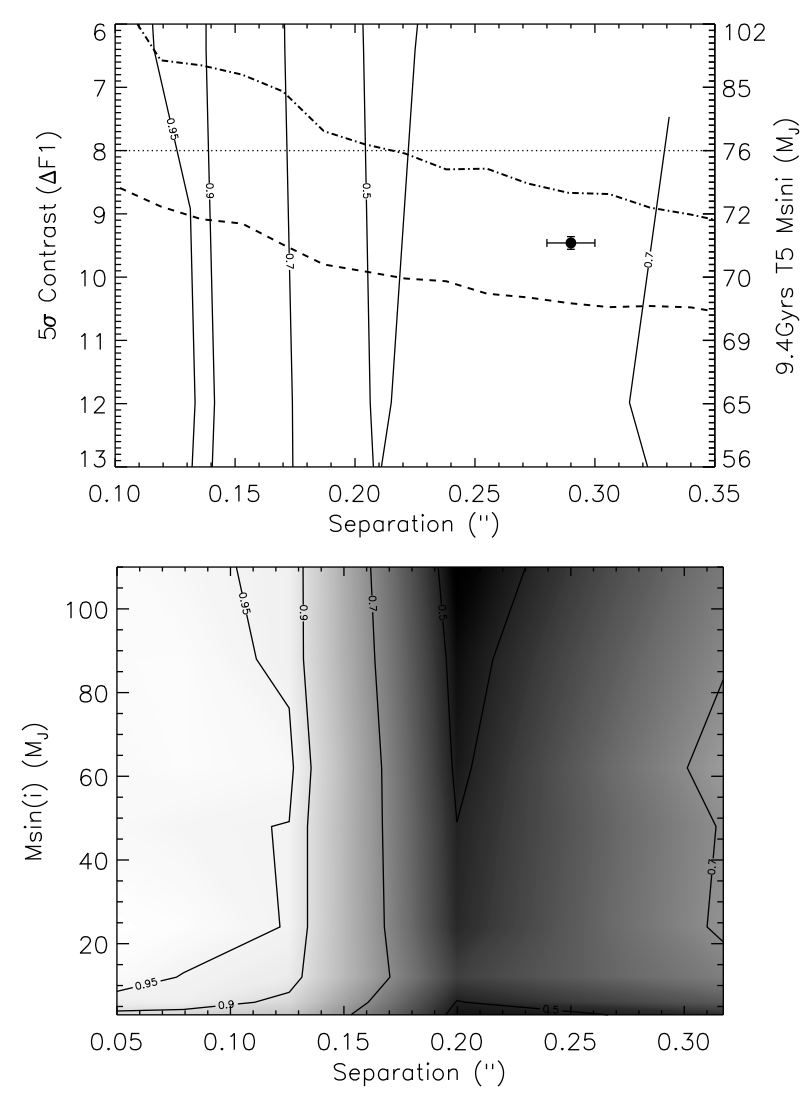

Fig. 13. The upper plot shows the contrast limits $(\Delta \mathrm{F} 1)$ for the star HD25874. A contrast of $10.5 \mathrm{mag}$ is reached at $0.3^{\prime \prime}$, which is the separation of the potential candidate. The solid curves mark the radialvelocity confidence limits. The right hand $y$-axis shows the expected mass for a T5 dwarf at the age assumed for these objects. The horizontal dotted line marks the strong methane boundary. The best fit above this limit is to aid the eye since no detections were made beyond this boundary. The lower plot shows the radial-velocity confidence limits in mass-angular separation space. The values inside all solid curves are the confidence percentages.

bright spot to the extreme left middle of the left panel and its counterpart in the corresponding position of the right panel. Along the projected angle there is also another bright and dark pair that could be separated by the roll angle and these are found at the ends of the inner arc. As these are so close and connected to the central star we believe these to be an artifact of the PSF subtraction, however worryingly since they are found projected along the same axis as the potential candidate detection they may signify that the detection is an artifact as well e.g. uncorrected residual trefoil in the image.

As mentioned, Fig. 13 (upper) shows the contrast limits that were determined for HD25874, highlighting both the conventional AO and the SDI reduced limits. For companion candidates such as the one here it is clear that the SDI reduction performs significantly better than conventional AO e.g. gain of $\sim 2.5$ mag at $0.2^{\prime \prime}$. The confidence limits in this figure show a lot of structure at separations reaching well into the imaging phase space. This is due to the large baseline ( $>4 \mathrm{yrs}$ ) of observations, even though they describe a liner system. $95 \%$ confidence limits are seen to rule out objects down into the planetary mass regime with angular separations below $0.14^{\prime \prime}$, depending on where we place the boundary between exoplanets and brown dwarfs, and we can rule out such objects up to separations of almost $0.18^{\prime \prime}$ at
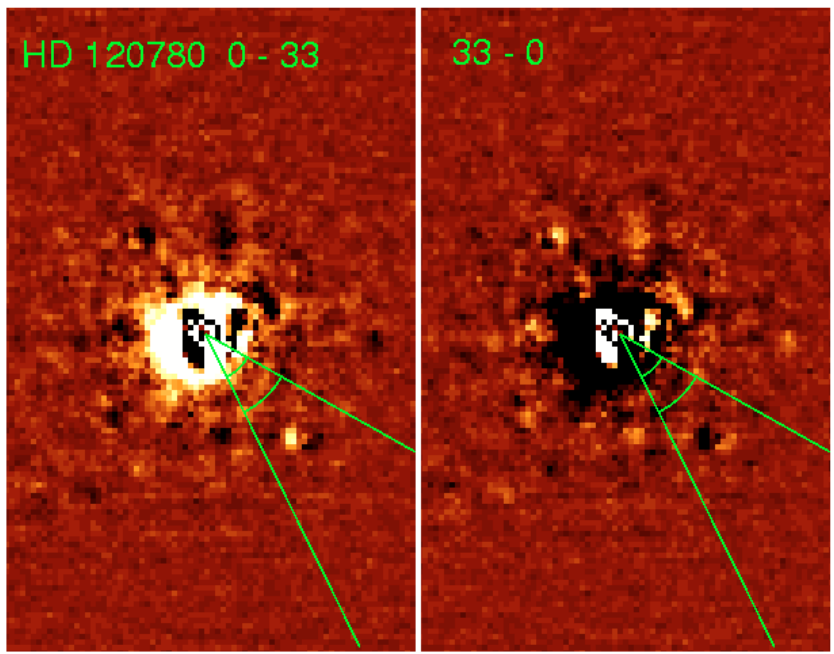

Fig. 14. The annotated image of the star HD120780 containing a possible detection of the companion object. The green lines mark the projected roll angle of $33^{\circ}$ of the camera to select possible candidates from speckle noise. The positive and negative pair for this potential detection clearly intersect the green lines. This lends weight to the potential for this to be a single object seen through two separate roll angles of the camera.

the $70 \%$ confidence limit. The lower panel better highlights the structure in detectability for this star and shows clearly that we have high levels of confidence out to the timeseries of this data set. The gray scale shows similar structure to that of HD145825 with a large inner region that is highly constrained, a dark unconstrained region around $0.2^{\prime \prime}$ separation from the star and then a growing lighter constrained region out to separations of $0.3^{\prime \prime}$. This time the constrained region at larger separations arises not from any curvature in the velocities but due to the overall span of velocity across the data set, showing that it is unlikely that lower mass companions at these orbital separations could give rise to this data set. By combining the imaging data with the radial-velocity data, we can say that with $70 \%$ confidence we can rule out almost all companions to this star with separations below $0.17^{\prime \prime}$ (4.40 AU). Again we conclude that the companion to this star is probably a widely separated, low mass $\left(<70 M_{\mathrm{J}}\right)$ and therefore really faint sub-stellar object. If such is the case then there is a fairly high possibility here that the object is a brown dwarf located in the brown dwarf desert (Grether \& Lineweaver 2006). Note that there were no other objects detected around this star out to orbital distances of $\sim 52 \mathrm{AU}$.

Figure 14 shows the annotated (33 ${ }^{\circ}$ roll angle) for the star HD120780. The angle is projected along the solid lines radiating from the central pixel. The brightest object in this image is found between the projected solid lines, however since no counterpart was found this is a residual super speckle and shows that such artifacts still remain after SDI reduction. The potential candidate around this star is found to reside at a separation of $0.30 \pm 0.01^{\prime \prime}$ from the central pixel with a $\Delta \mathrm{F} 1$ contrast of $\sim 8.8 \mathrm{mag}$, and is again well below the strong methane boundary. Significantly though, the separation is in agreement with the object found around the star HD25874. Also the position angle of the object is $240^{\circ}$ which again is in agreement with the find around HD25874. The fact that both the separation and position angle agree for the possible candidate objects around both HD25874 and HD120780 strongly indicate that these detections are actually artifacts inherent in the reduction procedure rather 
Table 7. Summary of results from the combined SDI and radial-velocity constraints for all stars.

\begin{tabular}{cccccccc}
\hline \hline Star & $\mathrm{AO}\left(0.5^{\prime \prime}\right)$ & $\mathrm{AO}\left(1.0^{\prime \prime}\right)$ & $\mathrm{SDI}\left(0.5^{\prime \prime}\right)$ & $\mathrm{SDI}\left(1.0^{\prime \prime}\right)$ & $R V 70 \%$ & $\mathrm{RV} \mathrm{90 \%}$ & $R V 95 \%$ \\
\hline HD25874 & $9.5-71 M_{\mathrm{J}}$ & $11.2-68 M_{\mathrm{J}}$ & $11.2-68 M_{\mathrm{J}}$ & $11.3-67 M_{\mathrm{J}}$ & $2 M_{\mathrm{J}}$ & $4 M_{\mathrm{J}}$ & $9 M_{\mathrm{J}}$ \\
$\mathrm{HD} 32778$ & $8.7-72 M_{\mathrm{J}}$ & $9.0-71 M_{\mathrm{J}}$ & $8.9-71 M_{\mathrm{J}}$ & $9.3-71 M_{\mathrm{J}}$ & - & - & - \\
$\mathrm{HD} 91204$ & $9.5 \mid 70 M_{\mathrm{J}}$ & $10.1 \mid 68 M_{\mathrm{J}}$ & $10.0-68 M_{\mathrm{J}}$ & $10.0-67 M_{\mathrm{J}}$ & $3 M_{\mathrm{J}}$ & $13 M_{\mathrm{J}}$ & $30 M_{\mathrm{J}}$ \\
$\mathrm{HD} 120780$ & $8.9-66 M_{\mathrm{J}}$ & $9.8 \mid 60 M_{\mathrm{J}}$ & $10.0-59 M_{\mathrm{J}}$ & $10.3-57 M_{\mathrm{J}}$ & $3 M_{\mathrm{J}}$ & $35 M_{\mathrm{J}}$ & $23 M_{\mathrm{J}}$ \\
HD145825 & $9.3-57 M_{\mathrm{J}}$ & $10.9-41 M_{\mathrm{J}}$ & $10.6-44 M_{\mathrm{J}}$ & $11.0-40 M_{\mathrm{J}}$ & $3 M_{\mathrm{J}}$ & $11 M_{\mathrm{J}}$ & $15 M_{\mathrm{J}}$ \\
\hline
\end{tabular}

Notes. Columns 2 and 3 show the AO reduced contrast limits and their associated T5 mass limits at the age of each star for angular separations of $0.5^{\prime \prime}$ and $1.0^{\prime \prime}$ respectively. Columns 4 and 5 show the same results but for the SDI reduction. Columns $6-8$ show the minimum mass that was ruled out at confidence levels of 70,90 and 95\% respectively. These masses were for all separations and therefore show only the lowest mass reached by the simulations and are not constrained within a separation limit. Note the lack of this data for HD32778 due to the limited radial-velocity data set.
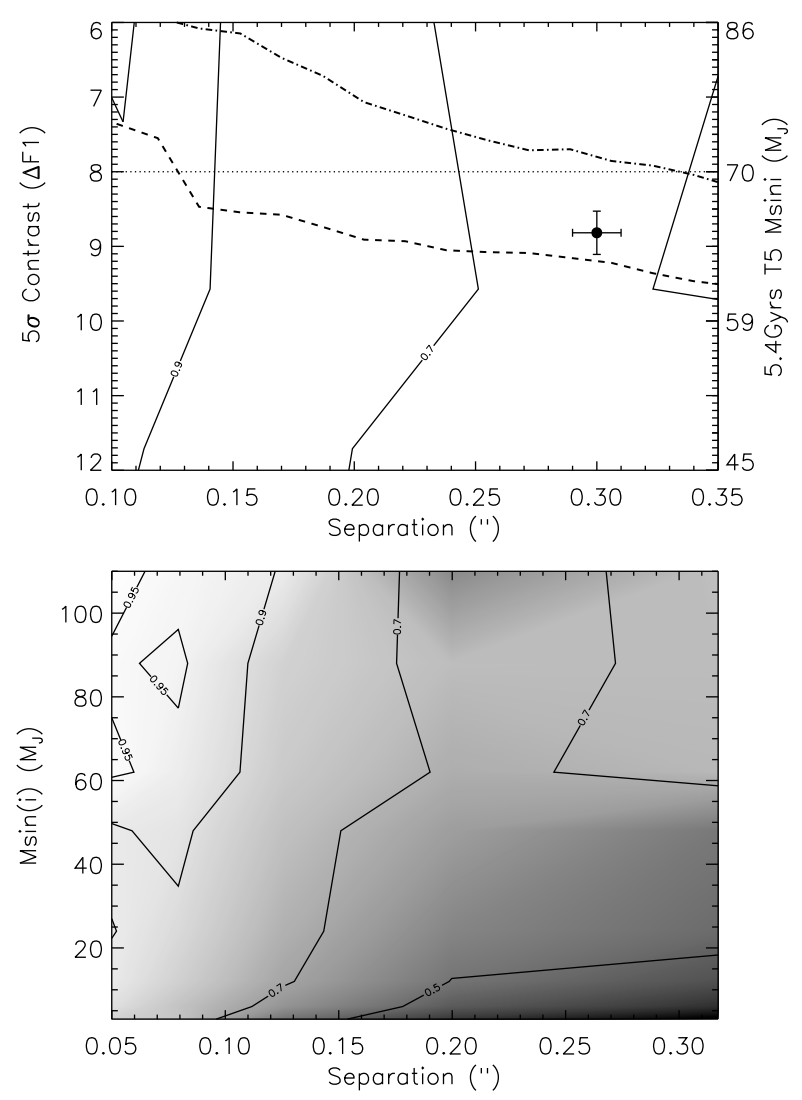

Fig. 15. The upper plot shows the contrast limits $(\Delta \mathrm{F} 1)$ for the star HD120780. An SDI reduced contrast of $9.3 \mathrm{mag}$ is reached at $0.3^{\prime \prime}$, which is the separation of the potential detection (filled circle). Note the large error bars on the contrast for this candidate since the contrasts were estimated using the other system fluxes since there were no unsaturated acquisition images for this star. The solid curves mark the confidence limits estimated from the radial-velocity timeseries. The right hand $y$-axis shows the expected mass for a T5 dwarf at the age assumed for these objects. The horizontal line represents the strong methane boundary. The lower plot shows the radial-velocity confidence limits in mass-angular separation space. The values inside all solid curves are the confidence percentages.

than an actual detections of the companion objects to both these stars, possibly spider arm residuals.

The contrast curves created for this system (Fig. 15 upper) are extrapolated from the unsaturated images of the other systems. The detection here is marked by the filled circle with associated uncertainties to highlight the contrasts achieved at such low angular separations. The detectability probability in this region is found to be 50-70\%, again showing that more data would be useful to better constrain the companion parameters, even at such low separations. The detectability region is better seen in Fig. 15 (lower) where again we have over 90\% confidence reaching out beyond $0.1^{\prime \prime}$ from the star and with fairly high brown dwarf masses, across the stellar mass regime. The gray scale in this region shows no real dark unconstrained regions, except for widely separated and low mass companions. Below the SDI limits we see that we can rule out lower mass companions with $90 \%$ confidence only out to separations of $\sim 0.14^{\prime \prime}(\sim 2.38 \mathrm{AU})$ at best. Yet above the $1 \sigma$ confidence level $(70 \%)$ we can rule out a range of lower mass companions reaching as far out as $0.25^{\prime \prime}(4.25 \mathrm{AU})$. Finally no other longer period methane rich companions were detected out to a separation of $\sim 34 \mathrm{AU}$.

\section{Summary}

We have performed a targeted direct imaging program to detect cool companions orbiting within $2^{\prime \prime}$ of their parent stars. The stars were drawn from the AAPS and Keck planet search projects and consist of objects that exhibit large radial-velocity variation over several years. These radial-velocities indicate the presence of a massive companion on a long period orbit. Five stars were examined with the NACO-SDI system on the VLT in Paranal, Chile. From the five, two possible detections were found around the stars HD25874 and HD120780. However, further analysis of these detections indicate they are probable residual artifacts since they are found to be located at the same distance from the central pixel and the same position angle in each of the images for each star. In addition we also present detectabilities for each system by analysing the radial-velocity information we have acquired. Each of these detections lie within sensitivity boundaries for these stars of between 50-70\%, meaning they could not be ruled out with any high degree of certainty from the radialvelocity data.

We have summarised the results of this work in Table 7, which shows both the broad-band AO and narrow-band SDI reduced contrasts and mass limits at separations of $0.5^{\prime \prime}$ and $1.0^{\prime \prime}$. Also the sensitivity limits from the radial-velocity data have been summarised at confidence limits of 70, 90 and $95 \%$ for each system, highlighting the minimum mass possibly detectable at each confidence level. The table shows the contrasts in magnitudes and mass limits in Jupiter-masses at $0.5^{\prime \prime}$ and 1.0" angular separations for AO and SDI images and shows the AO performs almost as well as the SDI at separations of $\geq 1.0^{\prime \prime}$. Also since the stars are fairly old the AO reduction tends to reach only a few Jupiter-masses above the SDI reduction for most of the sample, however when the star is fairly young (HD145825) the SDI 
reaches far deeper than $\mathrm{AO}$ i.e. $13 M_{\mathrm{J}}$ in this case. Also from this table we see that the radial-velocity confidence limits are all generally the same, apart from HD32778 due its limited number of data points and temporal coverage.

As the stars chosen are fairly old, a consequence of the radial-velocity selection method, the companions must be sufficiently massive to lie within the detection threshold of the instrument. Also since three of the five stars were found to have "liner" trends, and we expect these to be sufficiently massive, then we suspect either the uncertainty in age means we are underestimating our mass thresholds, the models are over estimating the magnitudes of the companions, the companions are aligned such that they are found behind, or very close to the central star $\left(\leq 0.1^{\prime \prime}\right)$ or the companions are sufficiently far out in the system that they are off the $2^{\prime \prime}$ contrast limit. A combination of the these mechanisms are probably at work. We find $5 \sigma(\Delta \mathrm{F} 1)$ contrasts of $11.5 \mathrm{mag}$ are possible using this method around bright $\mathrm{F}-\mathrm{K}$ type stars (and $5 \sigma \mathrm{H}$-band contrasts of $12 \mathrm{mag}$ for mid T-like objects). Such contrasts allow access to long period, massive $\left(\sim \geq 40 M_{\mathrm{J}}\right)$ methane objects for stars that typically constitute the bulk of radial-velocity programmes. In the future similar analyses as those employed here will lead to a greater understanding of the properties of exoplanets and brown dwarfs when extreme-AO systems can gain the contrasts necessary to directly image known planetary-mass companions detected by ongoing Doppler programs.

\section{References}

Allard, F., Hauschildt, P. H., Alexander, D. R., et al. 1997, ARA\&A, 35, 137 Baraffe, I., Chabrier, G., Barman, T. S., Allard, F., \& Hauschildt, P. H. 2003, A\&A, 402, 701

Biller, B. A., Close, L., Lenzen, R., et al. 2004, in Advancements in Adaptive Optics, ed. D. Bonaccini Calia, B. L. Ellerbroek, \& R. Ragazzoni, Proc. SPIE, 5490, 389

Biller, B. A., Close, L. M., Masciadri, E., et al. 2007, ApJS, 173, 143 Burrows, A., Marley, M., Hubbard, W. B., et al. 1997, ApJ, 491, 856 Butler, R. P., Marcy, G. W., Williams, E., et al. 1996, PASP, 108, 500 Butler, R. P., Tinney, C. G., Marcy, G. W., et al. 2001, ApJ, 555, 410
Butler, R. P., Wright, J. T., Marcy, G. W., et al. 2006, ApJ, 646, 505 Carpenter, J. M. 2001, AJ, 121, 2851

Chauvin, G., Lagrange, A.-M., Dumas, C., et al. 2004, A\&A, 425, L29

Close, L. M., Lenzen, R., Guirado, J. C., et al. 2005, Nature, 433, 286

Duquennoy, A., \& Mayor, M. 1991a, A\&A, 248, 485

Duquennoy, A., \& Mayor, M. 1991b, A\&A, 248, 485

Duquennoy, A., Mayor, M., Andersen, J., Carquillat, J. M., \& North, P. 1992, A\&A, 254, L13

Grether, D., \& Lineweaver, C. H. 2006, ApJ, 640, 1051

Henry, T. J., Soderblom, D. R., Donahue, R. A., et al. 1996, AJ, 111, 439

Jenkins, J. S., Jones, H. R. A., Tinney, C. G., et al. 2006, MNRAS, 372, 163

Jenkins, J. S., Jones, H. R. A., Pavlenko, Y., et al. 2008, A\&A, 485, 571

Jenkins, J. S., Jones, H. R. A., Goździewski, K., et al. 2009, MNRAS, 398, 911 Jenkins, J., Blundell, J., Jones, H., et al. 2010, MNRAS, submitted

Jones, H. R. A., Paul Butler, R., Tinney, C. G., et al. 2002, MNRAS, 333, 871

Jones, H. R. A., Butler, R. P., Tinney, C. G., et al. 2010, MNRAS, 403, 1703

Kalas, P., Graham, J. R., Chiang, E., et al. 2008, Science, 322, 1345

Leggett, S. K., Golimowski, D. A., Fan, X., et al. 2002, ApJ, 564, 452

Lenzen, R., Close, L., Brandner, W., Biller, B., \& Hartung, M. 2004, in Groundbased Instrumentation for Astronomy, ed. A. F. M. Moorwood, \& M. Iye, Proc. SPIE, 5492, 970

Liu, M. C., Fischer, D. A., Graham, J. R., et al. 2002, ApJ, 571, 519

Marcy, G. W., \& Butler, R. P. 1992, PASP, 104, 270

Marcy, G. W., \& Butler, R. P. 2000, PASP, 112, 137

Marcy, G. W., Butler, R. P., Vogt, S. S., et al. 2005, ApJ, 619, 570

Marois, C., Macintosh, B., Barman, T., et al. 2008, Science, 322, 1348

McCarthy, C., \& Zuckerman, B. 2004, AJ, 127, 2871

McCaughrean, M. J. 2003, Amer. Astron. Soc. Meet. Abstracts, 203, \#124.04

Meschiari, S., Wolf, A. S., Rivera, E., et al. 2009, PASP, 121, 1016

Mugrauer, M., \& Neuhäuser, R. 2005, MNRAS, 361, L15

Mugrauer, M., Neuhäuser, R., Mazeh, T., et al. 2006, Astron. Nachr., 327, 321

Mugrauer, M., Seifahrt, A., \& Neuhäuser, R. 2007, MNRAS, 378, 1328

Neuhäuser, R., Guenther, E. W., Wuchterl, G., et al. 2005, A\&A, 435, L13

Nidever, D. L., Marcy, G. W., Butler, R. P., Fischer, D. A., \& Vogt, S. S. 2002, ApJS, 141, 503

O'Toole, S. J., Tinney, C. G., Jones, H. R. A., et al. 2009, MNRAS, 392, 641

Rousset, G., Lacombe, F., Puget, P., et al. 2003, in Adaptive Optical System Technologies II. ed. P. L. Wizinowich, \& D. Bonaccini, Proc. SPIE, 4839, 140

Takeda, G., Ford, E. B., Sills, A., et al. 2007, ApJS, 168, 297

Valenti, J. A., \& Fischer, D. A. 2005, ApJS, 159, 141

van Leeuwen, F., \& Fantino, E. 2005, A\&A, 439, 791

Wittenmyer, R. A., Endl, M., Cochran, W. D., et al. 2009, AJ, 137, 3529

Wright, J. T. 2005, PASP, 117, 657

Wright, J. T., Marcy, G. W., Fischer, D. A., et al. 2007, ApJ, 657, 533

Wright, J. T., Upadhyay, S., Marcy, G. W., et al. 2009, ApJ, 693, 1084 